Article

\title{
Modeling Pulsed High-Power Spikes to Drive Piezoelectric Broad- band Transducers Improving SNR in Ultrasonic Imaging \& NDE
}

\author{
Antonio Ramos ${ }^{*}$, Abelardo Ruiz ${ }^{1}$, Enrique Riera ${ }^{1}$ \\ ${ }^{1} R \mathcal{E} D$ Group Ultrasonic Systems and Technologies. Institute ITEFI (CSIC). Serrano 144, 28006 Madrid. Spain. \\ *e-mail: aramos@ia.cetef.csic.es (AR)
}

\begin{abstract}
Ultrasonic imaging \& NDE applications can greatly improve their signal-to-noise ratios (SNR) by driving each transducer (composing piezoelectric arrays) with a spike giving pulsed power of $\mathrm{k}$-Watts, repetitively at a PRF $=5000$ spikes/s, by using a HV capacitive-discharge generator. However very-high levels, of pulsed intensities (3-10 A) and voltages (300-700 V) must be considered for a rigorous spike modeling. Even though the consumed "average" power will be small, the intensity through each transducer achieves several amperes, so the pulsed powers delivered by each HV generator can attain levels higher than in CW high-power ultrasonic applications: e.g., up to $5 \mathrm{~kW} /$ spike. This is concluded here from a transient modeling of the loaded generator. Then, unforeseen phenomena rise: intense brief pulses of driving power \& emitted force in transducers, and non-linearities in driver semiconductors, because their characteristic curves only include linear ranges. But fortunately, piezoelectric devices working in this intense regime do not show serious heating problems, because the average power remains being moderate. Intensity, power and voltage driving a broadband transducer from a HV capacitive pulser, are calculated to drastically improve (in $\cong 40 \mathrm{~dB}$ ) the ultrasonic net dynamic range available, with emitted forces $\cong 250$ Newtons $p$ and $\mathrm{E} / \mathrm{R}$ received pulses of $76 \mathrm{~V}_{\mathrm{pp}}$.
\end{abstract}

Keywords: efficient ultrasonic transceivers, broadband piezoelectric transducers, industrial NDE, medical imaging, pulsed high-power spikes, HV capacitive-discharge pulsers, high-current driving, high dynamic range.

\section{Introduction}

Each transducer channel (emitter \& sensor) involved in applications for ultrasonic imaging, using piezoelectric transducers, is usually classified as of low power, as it happens in systems developed for Industrial Non-Destructive Evaluation (NDE) and also in the imaging transducers employed for Medical Diagnosis applications (Echography). This power level classification is adopted because the total integrated power consumed (from the HV electrical supply) by each working ultrasonic channel is of the order of Watt, when it is integrated in its averaged value during one second (thousands of driving spikes).

In consequence, linear approaches are usually considered for the design, development and matching of transducers with driving pulsers, for ultrasonic imaging [1-4]. Nevertheless, in new very efficient ultrasonic detection \& imaging with high-resolution (HR), where high signal to noise ratio (SNR) is required, capacitive discharge schemes, inductively tuned [5-6], have to be adopted, because they are a direct way to obtain spikes with pulsed high-power, e.g. $1600 \mathrm{~W}_{\mathrm{pp}} /$ driving at only $400 \mathrm{~V}$, repetitively at typical pulse 
repetition frequencies $(\mathrm{PRF}=5 \mathrm{kHz})$. It is needed due to the low transducer motional resistance Rm (tens / hundreds of $\Omega$ ) and its rather high capacitance $(\mathrm{nF})$, for the usual inspected materials. This aim is possible with a limited size in driving electronic devices.

In a typical case, e.g., $R_{m}=150 \Omega$, the energy stored in a capacitor of $10 \mathrm{nF} \cong 0,8$ milliJoule, during $\cong 100$ useconds (with maximum charging current $<45 \mathrm{~mA}$ from HV supply, but only in first $\mu \mathrm{s})$, can be used for a sudden pulsed high-power wideband driving. This assures currents trough transducer of several Amperes / spike, during usual widths, 100$500 \mathrm{~ns}$ in Megahertz range, but with a final power consumed of only $\cong(800 \mu \mathrm{Watt} / \mathrm{spike})$.

In fact, an efficient driving with such a pulsed high-power, for the above mentioned high-SNR applications, implies some requirements of short duration across the transducer terminals: for instance, the supply of elevated pulsed currents from the output stages of the HV pulse generators, which can attain up to $10 \mathrm{~A}$ during hundreds of ns.

Such extreme circumstances are not normally considered in the design \& development of efficient broadband ultrasonic emitters. But it would be very convenient to include these pulsed aspects, as starting data, in accurate modelling \& simulation steps prior to design new ultrasonic imaging and NDE systems needing high signal-to-noise ratios.

In order to obtain a quantitative determination of the mentioned extreme aspects, a rigorous analysis must be made of some pulsed performances of the capacitive multi-generators to be employed for ultrasonic inspection. These appear during very short time intervals in each one of the repetitive cycles involved in each transducer driving regime of this particular type of designs for new HR industrial and medical imaging. And it should also be included the inductive parallel tuning and diodes involved, so that the second edge of HV spikes can be adjusted to a verticality similar to that of the first edge [5].

As other conditions, for the here analyzed ultrasonic systems, driving peak voltages in emission should be rather high, so assuring amplitudes bigger than usual (hundreds of $\mathrm{mV}$ ) in the received signals, to have final high performances. Also, the design of the whole ultrasonic transceiver must seek to improve the global SNR, so achieving echo-pulses with greater dynamic range than the current ones, for obtaining more clear measures \& images.

Fortunately, in new very efficient applications for ultrasonic imaging, the total average power delivered to each one transducer involved in PZT ultrasonic arrays, from HV capacitive pulse generators, could remain in a moderated value at the order of few Watts / transducer, for usual imaging PRF, by maintaining the supply HV voltage $<500 \mathrm{~V}$.

In spite of these limited values in driving voltage and consumed power, very-highpower phenomena of brief duration (hundreds of ns) could be observed by transient analysis under the pulsed regime imposed in ultrasonic imaging systems. So, the pulsed electrical power, delivered to each transducer by an efficient capacitive driver, in the usual repetitive driving with short spikes (at conventional PRF: 2-5 kHz), will attain similar levels than in a single transducer involved in CW high-power ultrasonic applications: for instance, up to $5 \mathrm{~kW}$ in pulsed power, during each short emission process performed for each one of the channels in typical efficient imaging applications using piezoelectric arrays.

Then, due to all the previously commented aspects, several intense transient phenomena will happen thousands of times per each second, during the practical behavior of 
new very efficient emission equipment employed for HR images forming: in particular, high-peak electrical intensities in all the electronic pulsers associated with an imaging array, and elevated peaks of driving power \& emitted force in the involved transducers. As a consequence of this, high-peaks (only temporary) in the involved parameters, and notable short-duration non-linear electrical / piezoelectric pulsed responses could appear, due to the associated pulsed regime in this type of efficient HR imaging \& NDE applications.

For instance, pulsed but important parameter variation ranges, would be experienced in efficient $\mathrm{HV}$ electronic drivers included in the ultrasonic imaging systems under study. Thus, some semiconductor devices included in these electronic pulsers for NDE can enter in the saturation state under pulsed intensities of up to 5-10 amperes. These so high levels could originate non-linear behaviors very different respect to those observed in the low electrical intensity cases. And the high electrical intensities and voltages (300$700 \mathrm{~V}$ ), supported by some components, could lead to quite surprising temporal electronic responses, which should be taken into account in the modeling and simulation stages, during the design of new equipment for very-efficient ultrasonic imaging purposes.

Really, these non-ideal and non-linear behaviors have not been expected in a broadband ultrasonic channel using low-power transducers, because the total consumed averaged power is rather small in these types of applications. But the pulsed ultrasonic energy repetitively emitted by the involved transducers, and the instantaneous electrical power IEP (in each pulsed shot) delivered by the electronic HV drivers (during each repetitive very-short emission time-period), can attain higher levels, even, than in typical CW highpower ultrasonic applications: for instance, several $\mathrm{kW}$ of instantaneous power delivered toward each emitter transducer can be registered during every individual driving.

For comparative purposes, for instance in power CW ultrasonic applications [8] it is usual to register, for the medium power cases, driving peak values as: $600-700 \mathrm{~V}$ in voltage, and 0,6-0,7 A in current; i.e., 400-500 W in power. And in the very-high power ultrasonic equipment, values like [1400-1800 V in voltage, 1-1,2 A in current, and 1,5-2 kW in power] can be attained across the electrical terminals of the working ultrasonic transducers.

As other indicative data necessary for understanding -a) why it results necessary to design special driving schemes based on HV capacitive discharges, and -b) the causes of the required pulsed high-power: it must be noted than the typical values of the input resistive impedances of the considered broadband piezoelectric transducers, when they are inductively tuned around their series resonances, usually range between 50 and $300 \Omega$, for efficient ultrasonic radiations in more usual media; and the capacitances across transducer terminals can attain several nF. Other HV generators, useful with high-impedance ultrasonic transducers, are not efficient options for driving these demanding load impedances.

As a summary of the aspects above described: some unexpected very-high values in pulsed regime, like maximum electrical levels supported by some electronics drivers and piezoelectric transducers, must be taken into account for a rigorous analysis of the whole emission piezoelectric process. This must be made under the specific data of pulsed power and electrical current needed for the efficient driving required, for instance, in ultrasonic imaging for medical and industrial NDE applications with high-resolution and SNR [7]. 
In the following sections, a detailed analysis is performed of the high-voltage driving, current intensity and supplied power, delivered from each involved HV electrical generator, during the repetitive short emission periods in efficient ultrasonic imaging systems and NDE for industrial quality control, with a backed transducer in $\mathrm{MHz}$ range.

Firstly, the typical capacitive scheme for high voltage / current driving is shown and analyzed. In order to illustrate its associated final high efficiency, some published author's experimental responses in through-transmission are analyzed for the $1 \mathrm{MHz}$ backed transducer, with a pulsed driving of only $\cong 310$ Volts, using the here considered intense excitations, based on HV capacitive discharges. These interesting results $[9,13]$ are explained: final intense broadband ultrasonic pulses with very-high amplitude, as high as 50-76 Volts pp, are received across the terminals of other similar backed transducer with its frequency band also centered around $1 \mathrm{MHz}$. This represents a very important improvement in SNR.

After this, several author's options for mathematical and circuital modeling of wideband piezoelectric emitters are described. They can be employed for numerical simulation of this type of capacitive drivers, in order to quantitative calculate (with different degree of accuracy) the abovementioned pulsed high-power behaviors, associated with those.

Some simulated and experimental pulses are shown for high-SNR emission cases. In this context, non-linear phenomena during the HV excitation process are commented, and very clear motional piezoelectric influences, from the transducer vibrations toward the HV pulse generators, are shown for a particular setting during an emission process.

Single spike-type driving waveforms as those here considered, are experimentally measured and simulated, in HV generators including components suffering non-linear behaviors. Nevertheless, non-linear risks, in the broadband signals finally received from the medium, are rather of moderate importance because in normal imaging applications a rather harmonic performance remains in their spectral E/R responses due to some filtering effects by the limited-bands of emitter and receiver transducers, not showing (in general) saturation phenomena in the emitted pulses, due to over-driving situations.

Finally, some obtained quantitative results will be discussed, in relation to pulsed high-power waveforms at the output of a tuned capacitive driving case with moderated voltage, applied to an emission ultrasonic application as those here analyzed. These results illustrate pulsed magnitudes being comparable to many cases of CW high-power applications, although the CW regime is not employed in imaging and NDE systems. The resulting force and velocity pulses over the transducer emission face, will be also shown.

\section{Systems and Methods}

\section{1. Electronic systems for high resolution \& SNR in ultrasonic imaging and NDE}

The efficient electronic equipment specifically designed for ultrasonic beam forming, during the images syntheses, widely employed in applications for -a) industrial non-destructive evaluation NDE for quality control of materials and b) high-resolution (HR) medical diagnosis- has the general block diagram detailed in the Figure 1.

These systems, designed to improve resolutions in the $\mathrm{MHz}$ frequency range, include many piezoelectric transducers and pulsed electronic channels for emission (pulsers) 
and reception (receivers) of very short pulses (<1 microsecond), which produce multiple echo-frames, in a parallel way. Both types of pulsed waveforms are electronically focused and scanned, firstly in the emission stage and posteriorly in the reception stage, by means of multiple precision delayer and Mux-DMux units of the electrical HV pulses and also of the corresponding echo-signals. The involved channels are dynamically controlled during each one of the successive signal acquisition cycles (around 5000 times per second).

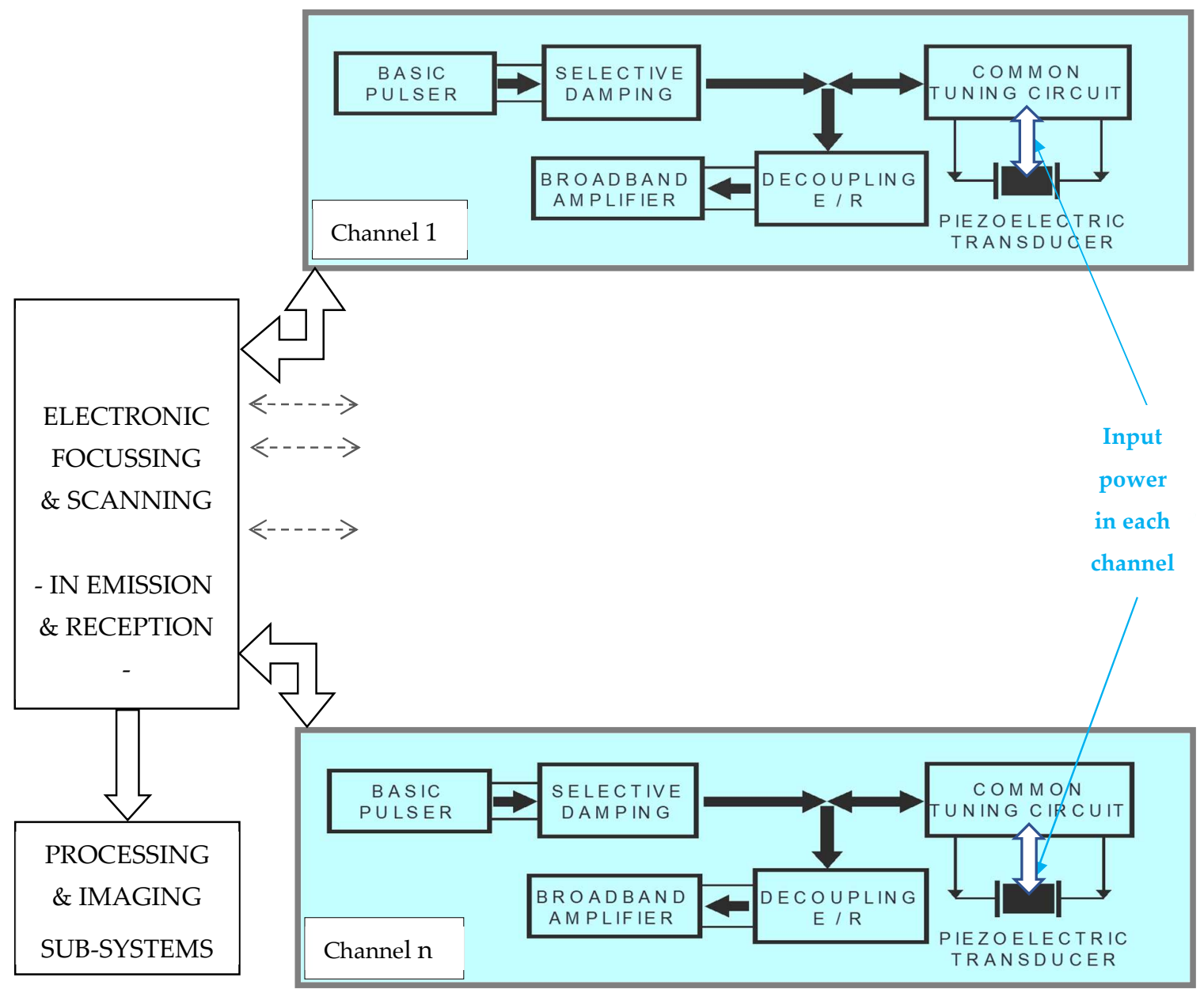

Figure 1. Structure of HR imaging systems, which includes an elevated number of controlled E/R ultrasonic channels (in general, between 32 and 256).

In each individual driving pulser, a selective damping block works in cascade with a basic H.V. pulse generation unit. The damping block notably reduces certain oscillations induced in driving pulses, due to the more usual emission matching circuits include an inductive tuning for medical and NDE imaging applications [13]. 
In all the parallel pulse-echo units of Figure 1, both piezoelectric sections share a unique matching circuit (damping and tuning), due to only one of the two sections is simultaneously working, well as an emitter or well as a receiver.

Logically, the periods with high values in pulsed power are those related to each repetitive HV driving. The excitation pulse amplitude, in voltage, are normally 300-500 Volts, but can attain up to 700 Volts. The pulse repetition frequencies (PRF) for pulse-echo imaging vary normally between 2.000 and 10.000 spikes / sg, depending on final imaging resolution (number of emitted foci and lines). The durations of each high-voltage driving spike, varies between 100 and 500 nanoseconds, in the more habitual inspection cases.

These electrical characteristics assure, in all the channels, an efficient transducers excitation repeated several thousands of times in each second, in order to obtain the elevated number of echo-signals that make possible the very fast processes employed nowadays for electronic focusing and scanning of the finally involved pulsed ultrasonic beams.

Each pulser typically includes a number of non-linear circuits, e.g., semiconductor devices $[5,16]$. These circuits have to support short pulsed intensities of up to ten amperes, and in consequence they can suffer a high-saturation state, with possible non-linear responses. In fact, the characteristics curves of many electronic devices contemplate only linear responses under moderate intensities values (e.g., <1 Ampere).

But, fortunately, the piezoelectric devices, working in such a high-power transient regime, do not show serious heating problems (which could be potentially destructive), because the associated mean power dissipation remains in the order of the Watt. However, very-high levels in both, pulsed electrical intensities (2-10 A) and voltages in the HV output stages (300-700 V), could lead to transitory non-linear behaviors, which must be considered in the imposed transient regime, for a precise modeling of the time and frequency response of each pulser stage [11-12].

\section{2. Circuits for high intensity transducers driving using HV capacitive discharges.}

The capacitive driving pulsers for efficient ultrasonic emission usually include highvoltage negative ramp generators, firstly proposed through a thyristor in [10] and after that through a Mos-Fet power transistor connected to a selective damping network [5]. In the second case, a very-low series impedance and short fall times are obtained. The ramp generator is connected in cascade with specific circuital networks [5] in order to achieve a good electrical matching with broadband emitter transducers and to finally attain highpower spikes for obtaining very high-intensity in the ultrasonic emission process.

Figure 2 shows the blocks diagram of an efficient driving of broadband transducers in a frequency range up to $30 \mathrm{MHz}$, with tuned narrow pulses of pulsed high power [7].

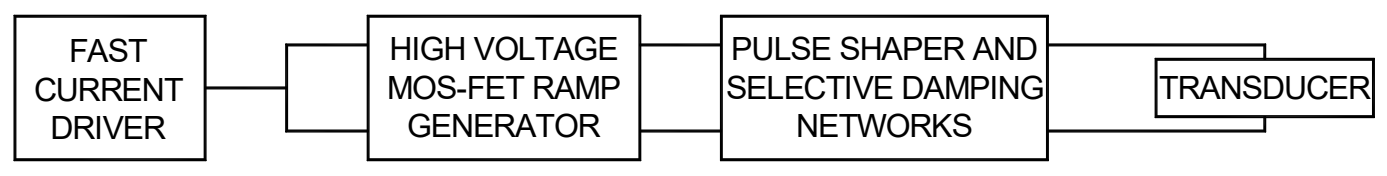

Figure 2. Block diagram of HV pulse generators for efficient driving of broadband transducers. 
The circuital topologies of the HV ramp generator and of the pulse shaper \& selective damping, depicted in Figure 2, are detailed in Figure 3.

The HV waveforms across the transducer $\mathrm{Z}_{\mathrm{T}}$ use to adopt the shape of a "spike" as it is shown in Figures 3.b and 4 for a broadband piezoelectric transducer centered at 1 $\mathrm{MHz}$ : a negative HV pulse with a rather vertical first edge (few nanoseconds) followed by other positive edge of rather short duration. The inductance $\mathrm{L}_{0}$ (and in some occasions the Zener diode $Z$ ) are added [5] because it can improve the final transit-time of the final driving pulse across the transducer terminals $\left(\mathrm{Z}_{\mathrm{T}}\right)$. The diodes network $\mathrm{D}_{1}-\mathrm{D}_{2}$ eliminates possible oscillations of considerable level that would be originated by C-Lo resonance effects, after the launching of the main HV spike waveform. Rc can be in the order of tens of $\mathrm{k} \Omega$.

Finally, the resistance $R_{L}$ of very low value $(1 \Omega)$, is added as an electrical protection under the high currents present in the output channel for HV driving.

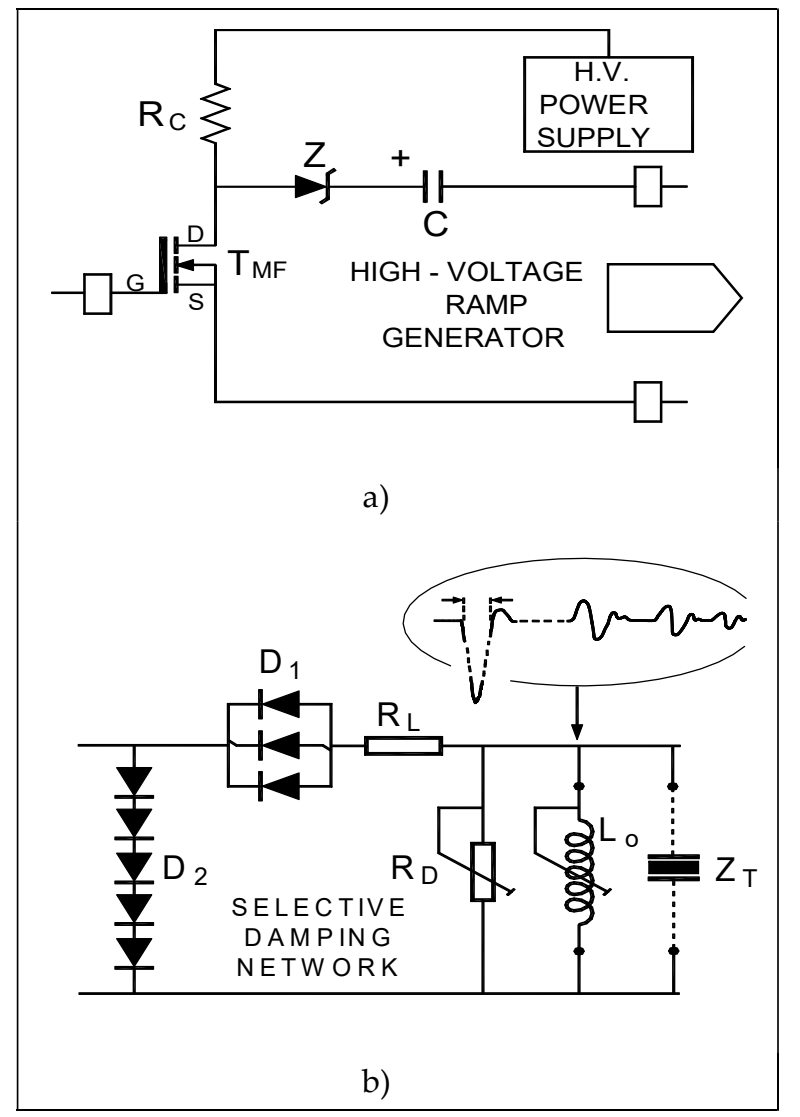

Figure 3. Circuital schemes for the blocks involved in the Figure 2: a) capacitive ramp generator and b) pulse shaper \& selective damping.

It must be noted that this capacitive circuital configuration offers the possibility to generate pulses of $\mathrm{HV}$, up to $500-700 \mathrm{~V}$, and high pulsed power (order of $\mathrm{k}$-Watt) across 
piezoelectric transducers in resonance conditions, even with very low resistive impedance (tens of ohms) and high capacitance in parallel (some nano-Farads).

It is usual, for ultrasonic imaging, to choose widths of spike between 100 and 500 nano-seconds, depending on the central frequency of the broad frequency band related to the input electrical conductance of each piezoelectric transducer to be driven.

Motional influences in a HV driving spike from the piezoelectric transducer

Using a short $\mathrm{T}_{\mathrm{MF}}$ saturation time, it is possible to observe influences in the electrical HV pulse (used for efficient driving) from the mechanical (motional) vibrations in the broadband transducer produced as response to the electrical excitation, such as it is shown in the measured waveform of Figure 4, for a $1 \mathrm{MHz}$ broadband backed transducer radiating in CFRP composite plastic material, and with this circuital setting: $T_{\mathrm{MF}}=\mathrm{IRF} 840, D_{i}=$ $1 \mathrm{~N} 4448, H$ supply $=250 \mathrm{~V}, \mathrm{C}=9,3 \mathrm{nF}, R_{\mathrm{L}}=1 \Omega, R_{\mathrm{D}}=100 \Omega$ and a turning off of the TMF MosFet transistor of $270 \mathrm{~ns}$ after the firing instant. These curious motional influences are related to the inverse piezoelectric effect produced in the driven transducer, immediately after applying the HV spike. This effect could be suppressed with a longer time "on" in the $\mathrm{T}_{\mathrm{MF}}$ device. Nevertheless, due to its short duration and time overlapping with the piezoelectric vibrations, it has not relevant consequences in the possible ultrasonic echo-signals received posteriorly in the same E/R ultrasonic transducer.

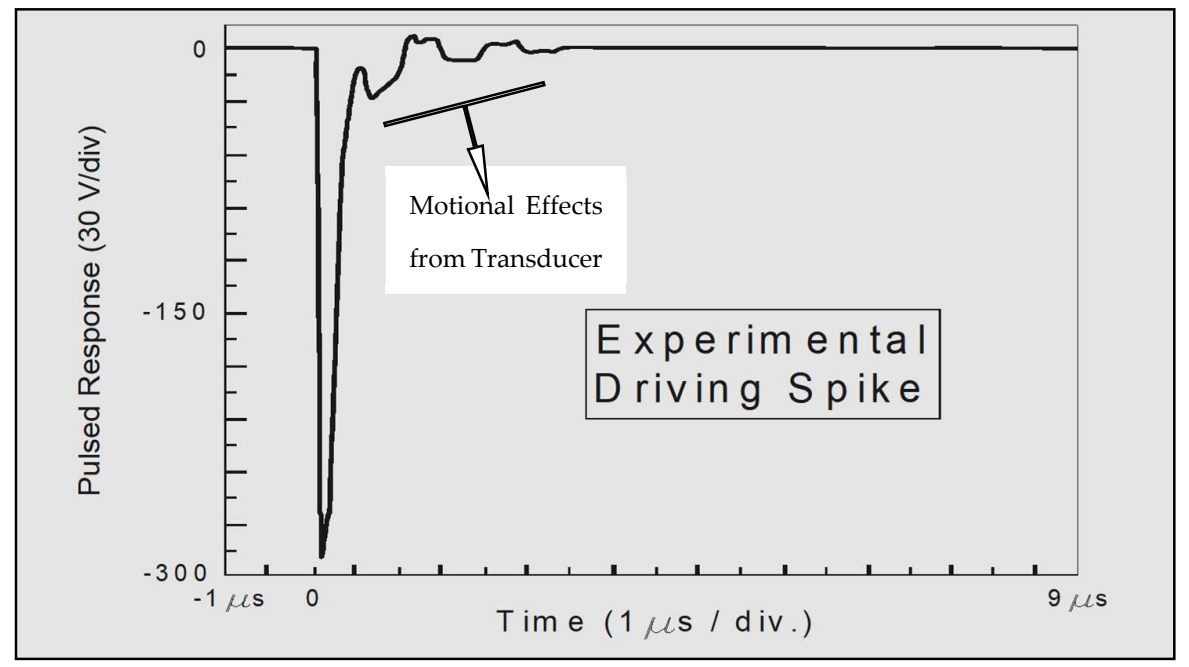

Figure 4. HV spike waveform for driving of a broadband piezoelectric transducer in the $\mathrm{MHz}$ range, showing mechanical influences from transducer vibrations, during first $3 \mu \mathrm{s}$.

The Figure 5.a), depicts the waveform for this specific transducer driving, calculated from circuital modeling as those explained in the following section. The non-linear mechanical influences from transducer vibrations in the HV spike also clearly appear, which have a similar shape to the emitting surface force, whose calculated waveform is shown in Figure 5.b). In fact, these electrically induced oscillations, during the first $4 \mu \mathrm{s}$, are in a close 
concordance with the behavior of the mechanical force registered in the face of the emitter transducer. An excellent agreement can be constated between Figures 4 and 5.a.
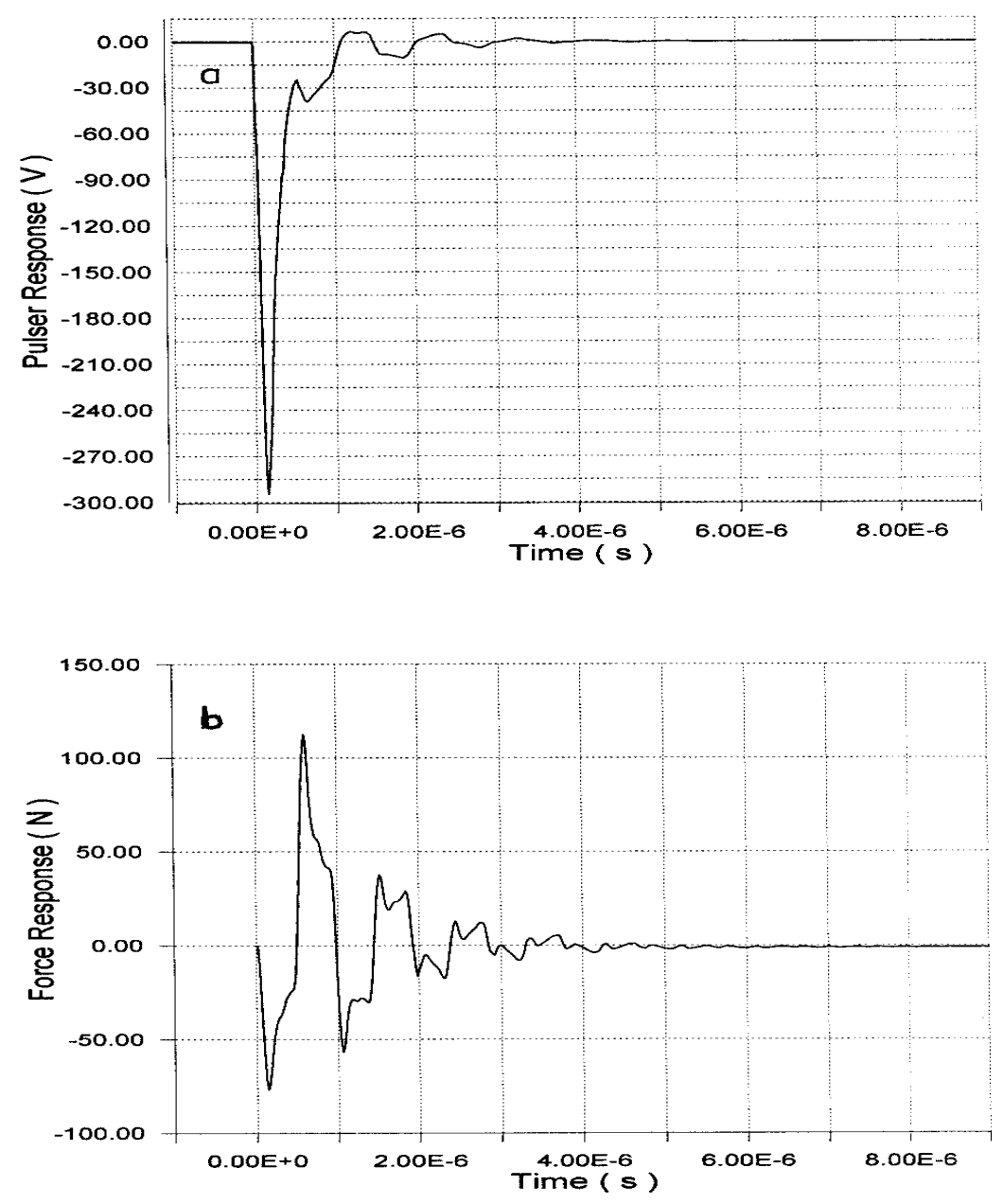

Figure 5. a) Computer simulation of the HV spike waveform for the transducer driving case of Figure 4, also showing the motional effects from the driven transducer, superimposed on the spike. b) Calculated transient force over the transducer radiating surface.

Improving reception SNR: The global efficiency of the electronic option, analyzed in this section for HV capacitive driving in emission stages, has a very important influence on the finally received ultrasonic signal values, in emission-reception NDE schemes. As an indicative example, the amplitude of the resulting broadband signals received by throughtransmission thru a $2 \mathrm{~cm}$ thickness methacrylate piece located between two broadband transducers, can attain values so high as 50-70 Volts peak-to-peak [9, 13], measured directly in receiver transducer terminals, without applying any type of reception amplifier.

In this referenced case, the two similar broadband piezoelectric transducers employed to obtain so intense received signals were the backed (6 Mrayl) models Tecal Q 269 / Q 270, constructed from a lead zirconate titanate piezo-ceramics with $\lambda / 4$ impedance matching layers [13]; $\mathrm{HV}$ driving parameters were: $\mathrm{V}=310 \mathrm{~V}, \mathrm{R}_{\mathrm{D}}=100 \Omega, \mathrm{C}=10 \mathrm{nF}, \mathrm{R}_{\mathrm{L}}=1 \Omega$. 
As values of reference, it must be noted that the usually reported pulsed echo-signals (received in similar ultrasonic applications) move around the range of hundreds of $\mathrm{mV}$.

This extremely advantageous results suppose a high SNR improvement, in the final $\mathrm{E} / \mathrm{R}$ broadband responses in the received stage (i.e., ultrasonic waveforms) $>37 \mathrm{~dB}$ respect to habitual values in similar ultrasonic situations. This is the main consequence of applying a high-efficiency electronic driving system of the tuned capacitive type, as those analyzed in this paper. In this case, an adequate inductive tuning of the employed transducers $(56 \mu \mathrm{H} / / 100 \Omega$ in emission and $22 \mu \mathrm{H} / / 1,5 \mathrm{k} \Omega$ in reception) was also an aspect of notable influence in the results, by a duplication of amplitude and bandwidth, simultaneously [9].

A priory, it is not an easy election to decide the more favorable selection of values for the coupling electrical components in the imaging \& NDE emission stages: damping resistance $\mathrm{R}_{\mathrm{D}}$, inductive tuning in emission $\mathrm{L}_{0}$ and discharge capacitor $\mathrm{C}$. In the following section, some modeling tools are described, very useful in order to assist in the design process of an optimum $\mathrm{HV}$ efficient driving system for each selected working transducer.

\section{3. Methods y Schemes for modelling and simulations of tuned HV capacitive drivers}

In this section, a description is made firstly of equations in time and spectral domains describing the driving voltage $D V$ from an inductively tuned HV capacitive driver.

By means of these expressions, a linear analysis could be made about the responses of this type of HV driver, using classical methods, but the presence of many non-linear electrical elements, in the circuital topology under study in this work, prevents in many cases the obtaining of driving waveforms as those encountered in the applications with real equipment containing the above described HV capacitive driver (Figure 3).

Analytic expressions in the time and spectral domains for the HV pulsed output generated by capacitive generators

Assuming a linear behavior in the electronic transmission stage of a channel as those involved in ultrasonic imaging, the spike for driving voltage $(D V)$ created by a capacitive driver could be expressed by a time convolution of an exponential function (created by the ramp generator in Figure 3) with the impulsive response $I R c c$ of the coupling network shown in this same figure:

$$
\text { Driving voltage }(t)=I R_{C C}(t) * V_{0} e^{-t / \tau}
$$

$\mathrm{V}_{0}$ and $\tau$ are the amplitude and fall-time, respectively, of a practical ramp function with a short fall time (10-15 ns), which has a time evolution near to an exponential curve.

And the finally received ultrasonic pulsed signals can be considered as a multiple convolution of the driving voltage (DV) appearing in (1) with the impulse responses of the successive emitting and receiving piezoelectric processes in a E/R transducer and those encountered through the diffraction and the propagation medium.

But, in many emission cases by using this mathematic way, a linear expression could be obtained, but only for the main part of $D V$ (Driving Voltage), just the first negative HV pulse $[5,7]$, and only by considering a simplification applicable uniquely in some cases. 
So, in some situations encountered in the practice, a possible linear option is to analyze this circuit in the frequency domain, taking into account that the effects produced by $R_{L}$ (a few ohms) and by the network of diodes $\mathrm{D}_{1}-\mathrm{D}_{2}$ in Figure 3, can be disregarded in principle, under certain conditions.

Then, a time response can be obtained [7] by means of the inverse Laplace transformation of the following analytical expression for $D V(s)$ :

$$
D V(s)=-C V_{o}\left[\tau C_{S} s^{2}+\left(C_{s}+\tau / R_{e q}\right) s+\left(1 / R_{e q}+\tau / L_{0}\right)+\left(1 / L_{0}\right) s^{-1}\right]^{-1}
$$

where: $C_{S}=C+C_{X T}$, being $C_{X T}$ the transducer static capacitance; and Req is given by:

$$
R_{e q}=R_{X T} R_{D} R_{S R}\left(R_{D} R_{S R}+R_{X T} R_{S R}+R_{X T} R_{D}\right)^{-1}
$$

In (3): $R_{D}$ is the electrical damping, $R_{X T}$ is the transducer resistive component in its motional branch under series resonance conditions ( $\left.1 / \mathrm{G}_{\mathrm{Max}}\right)$, and $R_{S R}$ is the input resistance of devices for display or amplification of the received ultrasonic signals.

From the inverse transform IFFT of the expression (2), an oscillatory waveform can be obtained in the time domain. But these high oscillations don't appear exponentially damped after the first half-cycle, as it happens in the practice due to the complex filtering effects of the diodes network $\mathrm{D}_{1} \& \mathrm{D}_{2}$ (after of the first big pulse in $D V$ ), have not been considered in this "linear" approach.

Nevertheless, now, in this point of the analysis, these effects could be just approximated, by emulating the cancelling (to putting at "cero") of the oscillatory response at the final of the first ascendant zero-crossing, just after the launching of the high-voltage spike, and so preventing an undesired lengthening on the driving pulse [9]. But this option is somewhat inaccurate in respect to the real experimental waveforms for tuned HV driving.

For obtaining a more accurate numerical simulation of this complex HV capacitive driving process, as needed here for an efficient quantization of the waveforms corresponding to the instantaneous electrical intensity and the pulsed power through the transduction stage, a non-analytical option must be employed, based in some circuital models containing the non-linear semiconductors under electrical high-intensities.

Some circuital solutions applicable for non-linear modelling and numerical simulations of tuned capacitive generators of pulsed high-power spikes

A practical solution for non-linear modelling of HV capacitive drivers is to apply software developed by authors, to implement and connect some PSpice circuital simulations of all the electronic/piezoelectric/ultrasonic subsystems involved, which use distinct equivalent circuits specifically created by us for the whole high-voltage emitters $[6,12,18]$. For instance, a quadratic approach can be implemented for the frequency dependence of mechanical losses in the piezo-electric transducer (XT), and non-ideal electrical effects can be considered [11, 16, 18]. These and other improvements are show in the following.

A first scheme including some non-ideal behaviors is shown in Figure 6, which are very useful to improve the modeling of $\mathrm{HV}$ transducer driving in the high frequency range (5-20 MHz), where parasitic inductances $L_{i}(i=1,2,3)$, associated to some printed circuit 
tracks, represent impedances that must be taken into account due to their possible filtering effects. These parasitic inductances are normally in the order of 50-150 $\mu \mathrm{H}$. L4-5 could be substituted by a transmission line if a coaxial cable was used for the transducer connection.

Di $(i=1 \ldots 6)$ represent the signal diodes that prevent the formation of harmful oscillatory signals, originated mainly from the series resonance between $C_{\mathrm{d}}$ and $L_{\mathrm{p}}$, and so allowing only the first half-cycle (the negative spike) to pass for transducer driving.

Other non-ideal effects are also considered, in this circuital modelling used for numerical simulations, with the explicit presence of the real effects of semiconductors ( $\mathrm{D}_{1}$, $\mathrm{D}_{2}$ and MFT) implied in the generic scheme of Figure 3, in some cases, even with their loss resistances under the high intensities (some amperes) in the electrical currents circulating through the principal output channels, for instance $\Delta Z_{\text {on }}($ few $\Omega$ ) related to series diodes D2-D6. $\mathrm{R}_{\text {in- }} \mathrm{C}_{\text {in }}$ represent possible effects of the input impedance of a display oscilloscope.

In addition, the mechanical losses in the piezoelectric stage of the emitter transducer [Rlosses $\left.\left(\omega^{2}\right)\right]$ are also considered in their real quadratic character. A block for the low-voltage pulsed control of the MOS-FET transducer MFT was included in this specific case. The stage for force emission (Fe) of the piezoelectric transducer can be represented by its Mason or KLM equivalent circuit incorporating quadratic mechanical losses [16, 18-20].

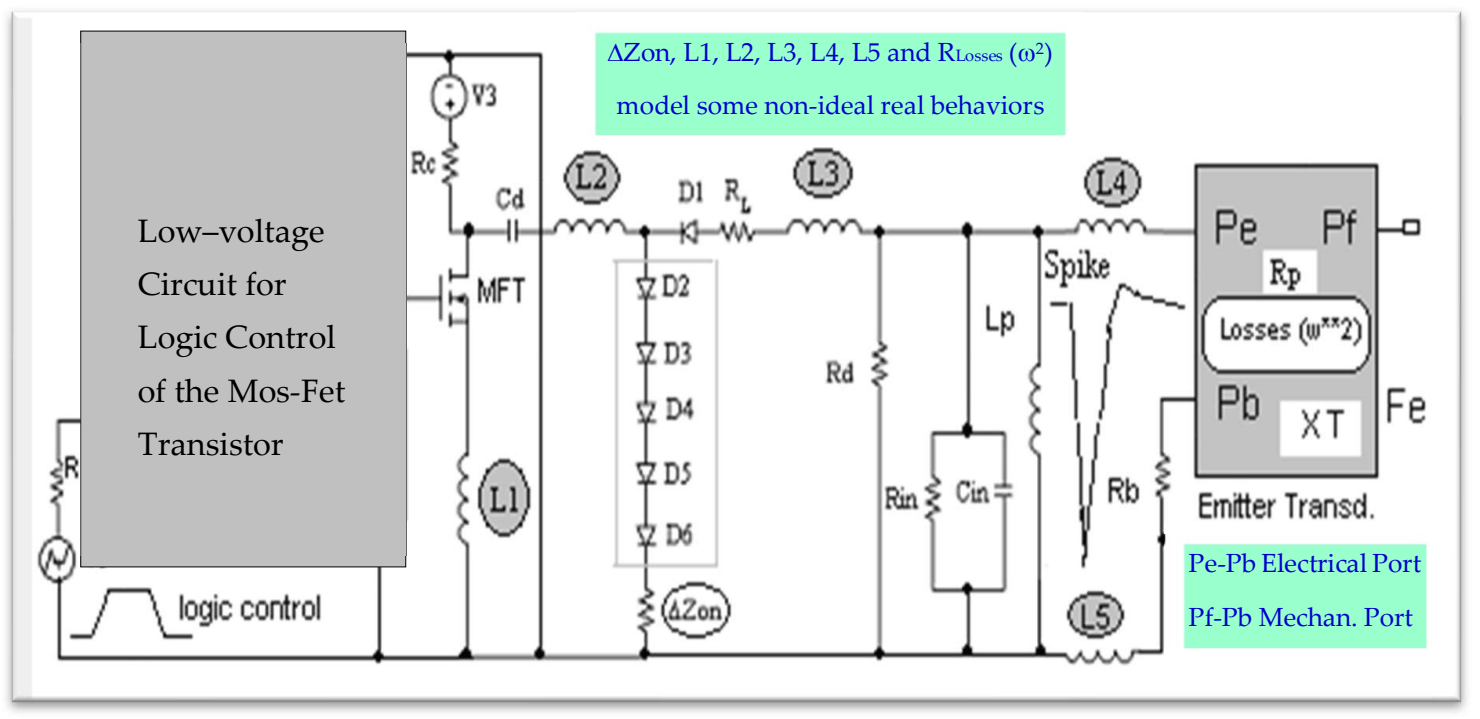

Figure 6. Scheme of a global circuital modeling for emission responses simulation under high-power capacitive driving in the high-frequency range, with electrical non-ideal behaviors and quadratic piezoelectric losses in XT.

Other simpler option applicable for non-linear modelling and simulation of capacitive generators of pulsed high-power spikes is shown in the Figure 7. It is specifically adequate for a lower working frequency ultrasonic range, from 0,5 to $4 \mathrm{MHz}$.

In this alternative circuital option, the electronic driving section, giving an excitation voltage $\mathrm{V}$, can be modeled by means of an $\mathrm{HV}$ ramp generator $\left(\mathrm{V}_{\mathrm{G}}\right)$ from a $\mathrm{HV}$ supply $\left(\mathrm{V}_{0}\right)$, and including the diode networks, SM1 and SM2, and the parallel combination (impedance $\mathrm{Zp}$ ) of the resistive and inductive tuned damping components ( $\mathrm{R}_{\mathrm{D}}$ and $\mathrm{L}_{0}$ in Fig. 3.b). 
In this circuital representation case of Figure 7, a PSpice modeling of both emission \& reception stages is included, by means of: i) a simple implementation of the HV spike generator connected to the E/R basic piezoelectric emitter sections, and ii) the circuital concretion of the emitted and receiver impedance matching layers to the propagation medium; this is taken into account by means of two transmission lines Tlayer 1-2 in Fig. 7.b. Here, Rf represents the acoustic impedance of the propagation media, and $\mathrm{Rb} 1$ and $\mathrm{Rb} 2$ are the acoustic impedances of the E/R transducers backing sections [14-15, 18].

This second option was utilized for obtaining the driving pulsed current \& power and the mechanical emitted waveforms in the time domain, related to a moderated highvoltage example chosen in this paper in order to illustrate the pulsed effects of high-power character, that can be observed in many cases involved in the capacitive-discharge driving stages included in efficient NDE \& ultrasonic imaging applications.

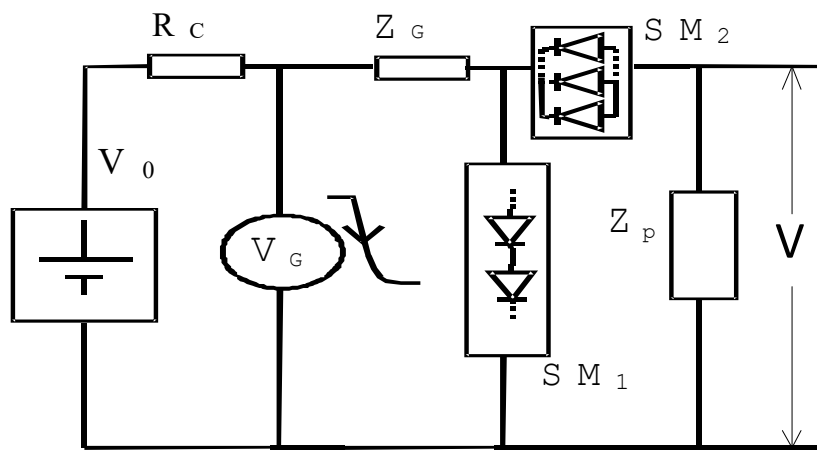

Equivalent $\mathrm{N}$ e tw ork of the H V D river

a)

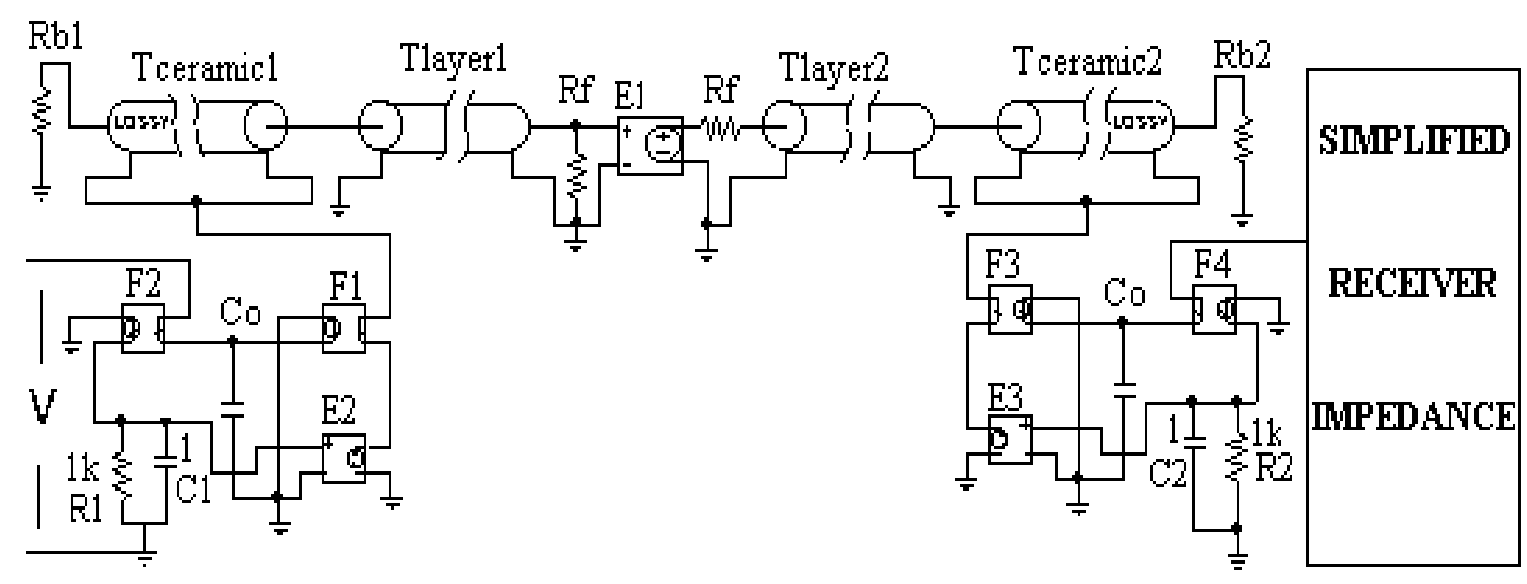

b)

Figure 7. Scheme of a practical option for circuital modeling of responses, in an intermediate frequency range, of ultrasonic transceivers under high-power capacitive driving considering piezoelectric losses in emission and reception. 


\section{Results in transient regime for the power outputs from a capacitive HV driver and resulting pulsed mechanical responses in a broadband piezoelectric transducer}

For making explicit indicative data related to a specific example of pulsed high-power capacitive-discharge driving, some selected pulsed results will be analyzed in the present section. Quantitative electrical waveforms involved in a high-efficiency generation of HV spikes will be shown, which could be classified as of an intermediate pulsed high-power, to repetitively drive a piezoelectric transducer, several thousands of times per second.

In order to obtain an accurate numerical time-simulation of this rather complex nonlinear high-power driving process (as it is needed to attain high signal-to-noise ratio in efficient NDE \& ultrasonic imaging), a precise electrical modeling in time domain (detailed in section II-3) was implemented in PSpice for the quantization of the driving waveforms.

Results are shown related to the pulsed electrical output intensity flowing through the transduction stage, and to the pulsed power delivered from a tuned HV capacitive pulser with a circuital scheme as that of Figure 3. These results were obtained by applying a non-analytical modeling, a necessary solution because the involved circuits contain several non-linear behaviors in some semiconductors, which can be described by means of explicit circuital models in moderate frequency range with piezoelectric losses (Figure 7).

This practical solution for the analysis, here employed to study the mentioned non -linear / ideal- responses, introduces a global circuital modelling of the involved electronicpiezoelectric-ultrasonic blocks. In this line, an implementation in the PSpice context was employed, from two coupled equivalent circuits with parameters related to this case.

A software specifically created by us, for the analysis of these pulsed high-power capacitive generators electrically matched to a broadband transducer, was specifically adapted. In this way, the numerical simulation $[12,18]$ of the overall driving-transduction sub-systems, involved in this particular application for transducer driving, was made.

Our practical objective was obtained by simulation and numerical calculation of a set of the more important waveforms produced in the loaded output of the capacitive high power pulser (as that shown in Figure 3), and of the subsequent mechanical pulsed waveforms radiation from the driven transducer to the propagation medium (across Rf in Fig.7).

These output waveforms are quite adequate for estimating the final efficiency associated to a typical broadband channel valid for efficient industrial ultrasonic diagnosis, that uses a piezoelectric transducer with a wideband centered around $1 \mathrm{MHz}$, and containing both parallel electrical tuning $\mathrm{L} 0$ and electrical damping RD. Figure 8 shows calculated curves for: driving current (a), instantaneous power b), supplied by the capacitive-discharge pulser to the transducer Tecal-Q 269 for a typical ultrasonic channel in NDE, with: $\mathrm{V} 0=450 \mathrm{~V}, \mathrm{Cd}=9,3 \mathrm{nF}, \mathrm{RD}=470 \Omega, \mathrm{RL}=1 \Omega$, and $\mathrm{L} 0=56 \mu \mathrm{H}$. The resulting emitted pulsed force (8.c) and velocity (8.d) in the emission face of the transducer are also shown.

The main part of the pulsed waveforms obtained for the time evolution of the electrical current and power delivered to the broadband transducer, have a duration near a 
half-cycle of the series resonance frequency $(1,02 \mathrm{MHz})$,corresponding to the maximum point of the spectral behavior of the transducer electrical conductance [9].

The electrical pulsed power delivered by this parameter setting of the HV capacitive generator, during their repetitive transducer driving, show levels typical of the CW highpower ultrasonic applications. These results confirm the main start point of this paper.

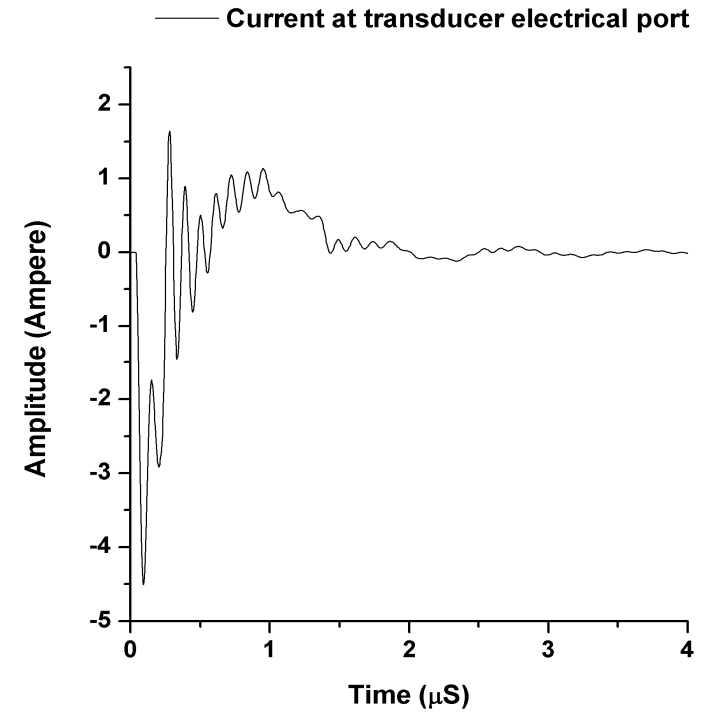

a)

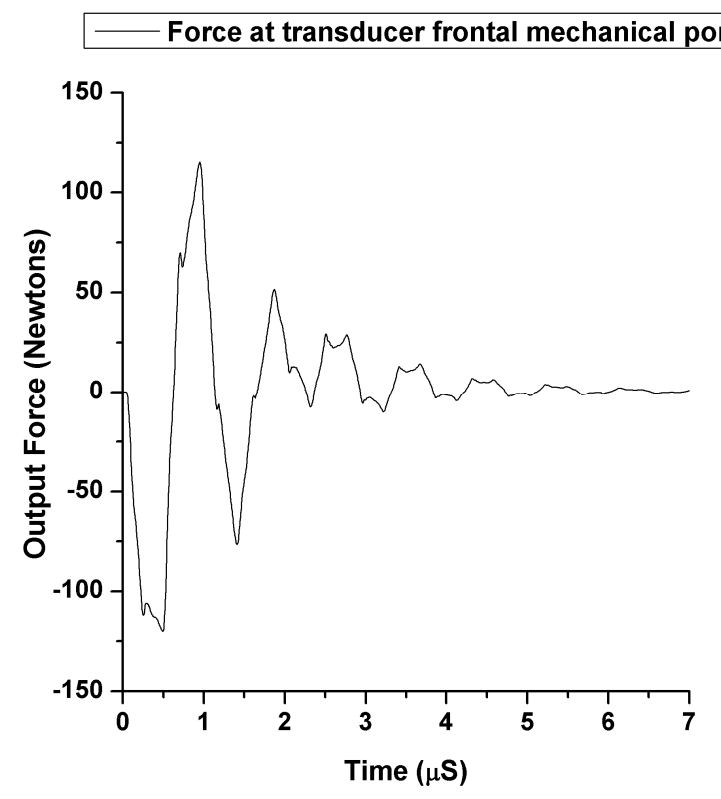

c)

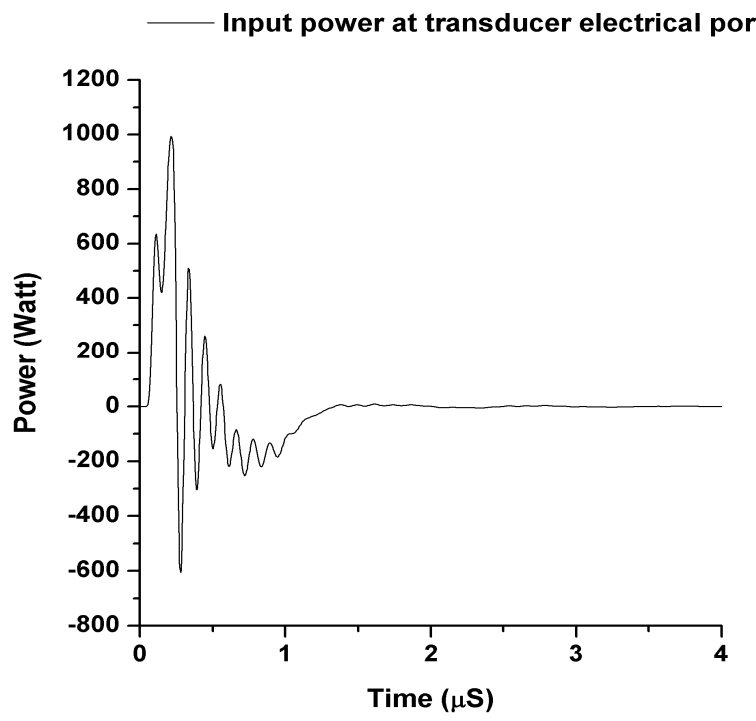

b)

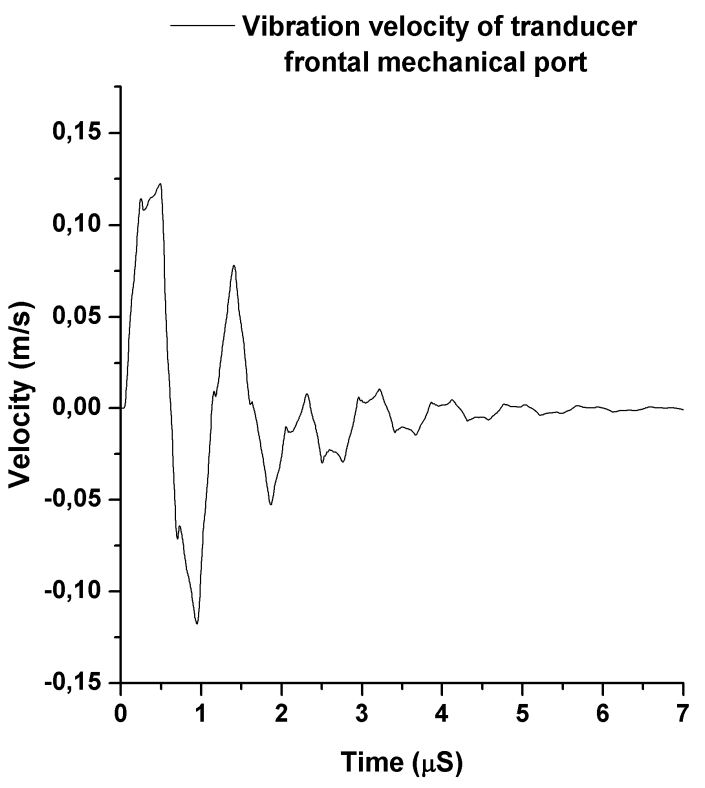

d)

Figure 8. Current (a) and power (b) waveforms in the HV pulser output calculated for the tuned HV capacitive driving of the Q 269 transducer, by using the circuital model of Fig. 7. Resulting force (c) and velocity (d) pulses calculated in the emission face of the transducer loaded with methacrylate. 
In the following and last paper section, the consequences of the above depicted graphic results are discussed, in relation to the possible obtention, for ultrasonic NDE and imaging applications, of high amplitude received signals with high SNR from the driving spikes with pulsed power of k-Watts. Also, some additional details, around the modeling \& simulation methods employed for the calculations, are specifically analyzed. The final advantages of the here analyzed tuned HV capacitive-discharge driving (for transducers with low and medium input impedances) over others circuital topologies for HV pulsers, are also specified and commented, including some generators designed for the excitation of high-impedance transducers non demanding so high electrical currents.

\section{Discussion and Conclusions}

From the analysis of the waveforms shown in the Figure 8.a,b, it can be appreciated the obtained electrical results during the driving of a piezoelectric mono-transducer, by means of an HV capacitive-discharge pulses generator, in this case with only $400 \mathrm{~V}$ output spikes but giving a pulsed power of 1.600 Watts pp and an electrical current of several Amperes. This constitutes a useful confirmation example showing some temporal high-power behaviors present in applications for efficient ultrasonic imaging and industrial NDE, classified in principle as working in the low-power range.

In consequence, the establishment of a pulsed high-power repetitive regime with a finally low averaged energy consumption, for several thousands of shots per second, has been confirmed for a broadband transducer in the ultrasonic range of the $\mathrm{MHz}$, as it was mentioned in the presentation and introduction of this paper.

The calculated waveforms were obtained by means of a careful modeling of an application designed for an efficient ultrasonic emission process, based on the generation of moderated high-voltage spikes, proposed by us for repetitive transducer driving, based on successive HV capacitive discharges (at a PRF $=5 \mathrm{kHz}$ ) over an inductively-tuned load connected to its output terminals, which contains the emitter transduction subsystem.

The results of the Fig. 8.a,b were attained from a global circuital modeling of an emission process, just one of those detailed in section II.3. In that part, firstly, a mathematical modeling approximation based on an analytical expression in the Laplace domain was detailed for these HV driving spikes; from this expression a time response can be obtained by applying the inverse Laplace transform. The finally so calculated driving waveforms are valid for many ultrasonic applications, but not when an efficient inductively-tuned scheme is introduced, in which case the modeled driving waveform only reproduces with accuracy the main part of the driving pulse, i.e., uniquely the initial negative spike.

But, by applying some circuital transient models (using equivalent circuits here proposed by us for the HV pulser and the tuned emitter transducer), the complete time-evolution of all the pulser output parameters (and their associated time-waveforms) can be calculated and graphically represented, around the series resonant frequency of backed broadband transducers coupled to the radiated plastic medium with an impedance matching layer.

It was confirmed that despite the pulsed power calculated across the analyzed transducer terminals ranges around k-Watts, fortunately, the consumed "average" power, under 
the common PRF used in imaging cases, was of only a few Watt. This very intense pulsed regime, during all the necessary electrical driving cycles in these types of E/R applications, was performed for an elevated pulse repetition frequency (the typical in imaging), and also for other additional experiments working at a bigger PRF of $10 \mathrm{kHz}$.

As additional electrical data referred to the pulser output parameters, in the here studied particular HV driving case, the pulsed electrical current flowing through the emission transducer attained 6 amperes $_{\text {pp }}$ during each electrical driving with output voltage pulses rather of moderated value, near to 400 Volts.

The application of non-linear modeling [11, 18] and associated simulation software $[6,12]$, both developed by the authors, for obtaining the electrical parameters values in the HV driving outputs and the pulsed mechanical waveforms emitted by the transducer (here considered for creating the pulses depicted in Figure 8), has proven that those computer tools are adequate to accurately represent the effects of an intense electrical excitation. In addition, the subsequent improved emitted waveforms from the transducer face (force and vibration velocity) were also calculated for this efficient driving (including non-ideal effects), being these simulated waveforms coherent with previous typical data.

High-power phenomena were detected and accurately quantized, with elevated pulsed values for the electrical current and power parameters in the emission stage of this typical mono-channel ultrasonic NDE application working in broadband conditions, with a central series resonant frequency around $1 \mathrm{MHz}$ under the usual transient regime.

But, in spite of the high-intensities and non-linearities related to the involved semiconductors (MosFet transistor and several signal diodes, included in the output electrical circuits), these devices, under this repetitive temporary high-power driving, have not shown serious heating problems, because, though the instantaneous power passing through them is elevated, during hundreds of nanoseconds, the total average powers dissipated in these electronic components really are quite small.

The new high-power generators, to be employed for high SNR ultrasonic NDE and HR imaging of inner parts in the inspected pieces, must be carefully designed for an adequate driving of each efficient PZT piezoelectric transducer with brief pulses having power enough to achieve high dynamic ranges in the finally acquired signals in reception. This high power is needed by the low working input resistance (tens or hundreds of ohms) of these tuned broadband transducers in $\mathrm{MHz}$ range; this resistance is the electrical consequence (in transducer terminals) of Rmotional in the mechanical branch, when a transducer radiates in its series resonance (i.e., at its frequency of maximum electrical conductance).

To solve this strong requirement in PZT transducers needs of high-power capacitive generators, as those here analyzed, providing short output pulses with maintained high electrical currents (up to 5-10 A), through inductively tuned transducers in working conditions, i.e. mechanically loaded with specific acoustic impedances of several MRayls. In fact, other HV generators of pulses up to $1000 \mathrm{~V}$, designed to be useful with air coupled transducers [21] or purely capacitive loads [22], are far from being able to electrically drive such high consuming NDE \& imaging transducers as those here considered (when they 
are loaded with solid or liquid media), during the needed hundreds of nanoseconds in each HV excitation at PRF of several thousands of times per second.

Paper results confirm the special adequation, of this type of pulsed high-power capacitive-discharges driving, to the very-efficient broadband applications here considered. In particular, the achievement of signal-to-noise ratios (SNR) and related dynamic ranges as high as those investigated here (for both ultrasonic emission and reception processes) can be illustrated from the following data and conclusions found along our work:

- By using the here analyzed capacitive high-power pulsed excitation (with a rather moderated spike voltage of $400 \mathrm{~V}$ ), the resulting force emitted for the selected backed transducer (with $\lambda / 4$ coupling layer to methacrylate materials) attains a value of 250 Newton $_{\mathrm{pp}}$ in its radiating face, as it was shown during the first cycle of the emitted short pulse.

- As a consequence of this notable force level promoted by the capacitive driving option, it is possible to easily radiate acoustic fields (over all the aperture projection), with average values in the order of the MPascal pp by using simple unfocused transducers.

- The driving of the broadband transducer employed in this paper, with the here designed high-power spikes, made it possible to obtain received ultrasonic waveforms (in a E/R application) having short-time duration and with very-high amplitudes: e.g. $76 \mathrm{~V}_{\mathrm{pp}}$ directly at receiver transducer terminals, in a through-transmission (TT) regime through a methacrylate plate. This supposes improvements in SNR of up to $38 \mathrm{~dB}$ (without using any type of amplification, averaging or filtering), respect to the received signals in classical ultrasonic TT and pulse-echo imaging \& NDE applications inside of solid or liquids media.

And this SNR improvement could be extended 4,9 dB more by using spikes of $700 \mathrm{~V}$, when this voltage can be electrically supported by the driven broadband transducer.

- Thus, from all the quite efficient results above shown, it is possible to predict that the circuital topology, analyzed in this paper, can originate pulsed high-power driving spikes, with low output impedances (1-2 $\Omega$ ), very adequate for an efficient excitation of broadband piezoelectric transducers, being able to improve the final obtained SNR in broadband applications. This implies a possible extension of the net dynamic ranges available in current ultrasonic imaging \& NDE ( $\cong$ ranging between 30 and $70 \mathrm{~dB}$ ) up to $>70$ and $110 \mathrm{~dB}$.

This drastic improvement could be decisive, particularly for cases with high attenuations or low acoustic impedances in some inspected media: lung, wood, foam, cork, benzene, gases, etc., where e.g. losses of up to $74 \mathrm{~dB}$ are registered along only the 2 short internal paths between emitting and receiving faces of the matched PZT ceramics and the medium. In addition, internal piezoelectric losses and medium attenuation should be added.

As a final general conclusion, this paper has shown for high-power capacitive spike generators driving broadband transducers, that though the finally consumed "averaged" power (for all the necessary thousands of spikes during a second, in ultrasonic imaging) can be very small (a few watts), the repetitive pulsed intensities through the transducer can attain several amperes during each spike with rather moderate peak voltages $(400 \mathrm{~V})$. In consequence, the delivered pulsed power from this type of $\mathrm{HV}$ generators can arrive to levels typical in CW high-power ultrasounds: in particular, driving spikes with pulsed 
power of $1,6 \mathrm{~kW}$ pp, or even quite higher values (for spike voltages $>400 \mathrm{~V}$ ), could be generated in loading conditions, at typical high-repetition transducer driving ( $\geq 5000$ shots/s). This is a very favorable start-point in order to can obtain elevated dynamic ranges in the received signals during new practical situations for $E / R$ ultrasonic echo-detection, having very high attenuation through the propagation medium or strong decoupling with it.

Acknowledgements. Authors gratefully acknowledge the support of the R\&D projects: Spanish National Plan project RETOS DPI 2017-90147-R (Low intensity ultrasounds for early detection and modulation of tumor and stroma), and Ibero-American Network CYTED-DITECROD-218RT0545 (New noninvasive ways for early diagnosis of chronic and degenerative diseases).

Author Contributions: Initial Planning \& Conceptualization, A. Ramos. Modeling, A. Ramos. Software Simulations, A. Ruiz. Data curation, E. Riera. A. Ramos, A. Ruiz. Formal analysis, A. Ramos. Funding acquisition, E. Riera. Investigation, A. Ramos and E. Riera. Methodology, A. Ramos. Resources, A. Ramos. Software, A. Ruiz. Supervision, A. Ramos; Validation, A. Ramos. Writing- original draft, A. Ramos. Writing - review \& editing, A. Ramos. Electronic systems \& Experimental measurements, A. Ramos. Power Ultrasonics, E. Riera.

Conflicts of Interest: The authors declare no conflict of interest.

\section{References}

1. R. N. Thurston. Effect of electrical and mechanical terminating resistances on loss and bandwidth according to the conventional equivalent circuit of a piezoelectric transducer, IRE Trans. Ultrason. Engin. , Vol. UE-7, pp 16-25, 1960.

2. G. Kossoff. The effect of backing and matching on the performance of piezoelectric ceramic transducers. IEEE Trans. Sonics and Ultrasonics, Vol. SU-13, No 1, pp. 20-30, 1966.

3. L. Filipczynski. Transient equivalent circuit and negative capacitance of a piezoelectric transducer performing thickness vibrations, J. Techn. Phys. 16 (2), 1975, pp. 121-135.

4. G. Hayward. The influence of pulser parameters on the transmission response of piezoelectric transducers, Ultrasonics 23 (1985), pp. 103-112.

5. A. Ramos, P.T. Sanz and F.R. Montero. Broad-band driving of echographic arrays using 10 ns - $500 \mathrm{~V}$ efficient pulse generators, Ultrasonics, Vol. 25 1987, pp. 221-228.

6. Ruiz, A. Ramos, I. Bazan. Influence of the capacitive energy with distinct inductive tuning and damping on the merit figure of highvoltage driving spikes in medical applications for new ultrasound diagnoses. Conference: 2016 Global Medical Engineering Physics Exchanges (GMEPE/PAHCE-IEEE). pp 1-6. April 2016. DOI: 10.1109/GMEPE-PAHCE.2016.7504657. Corpus ID: 39776899.

7. A. Ramos and J.L. San Emeterio. Ultrasonic systems for non-destructive testing using piezoelectric transducers. Electrical responses and main schemes. Chap. 16 in book "Piezoelectric Transducers". Berlin, Springer-Verlag. (ISBN 978-3-540-77507-2), 2008, pp. 413-431.

8. J.A. Gallego, G. Rodríguez, V.M. Acosta, E. Riera, A. Cardoni. Power ultrasonic transducers with vibrating plate radiators. Chapter 7, in book: "Power Ultrasonics. Applications of High-Intensity Ultrasound”. Woodhead Publishing, Cambridge, UK. 2015. pp 159-193.

9. A. Ramos, J.L. San Emeterio, and P.T. Sanz. Improvement in Transient Piezoelectric responses of NDE Transceivers Using Selective Damping and Tuning Networks, IEEE Trans. Ultrason., Ferroelect., \& Freq. Cont, Vol. 47, 2000, pp. 826-835.

10. J.G. Okyere, A.J. Cousin. The design of a high voltage SCR pulse generator for ultrasonic pulse echo applications. Ultrasonics, Vol. 17 (1979), pp. 81-84. 
11. A. Ramos, A. Ruiz, P.T. Sanz, J.L. San Emeterio. Some non-linear aspects of the electronic stages in time-domain modelling of NDE pulse-echo ultrasonic systems. Ultrasonics, Vol, 40, 2002, pp. 253-258.

12. A. Ruiz, A. Ramos, J.L. San Emeterio, P.T. Sanz. Pspice simulation of transient responses of transducers and spike generators included in E/R ultrasonic systems. Acta Acustica united Acustica, vol. 88, Supp 1:S 1-165, 2002. Special issue Rev. Acústica, Vol. XXXIII, pp. ult04016 1-6, 2002. And CD Rom Forum acusticum (ISBN 84-87985-06-8).

13. A. Ramos, J.L. San Emeterio, P.T. Sanz. Electrical matching effects on the piezoelectric transduction performance of a through-transmission pulsed process. Ferroelectrics Vol. 202 (1). pp. 71-80. October 1997. DOI: 10.1080/00150199708213462

14. A. Püttmer, A.P. Hauptmann, R. Lucklum, O. Krause, B. Henning. SPICE model for lossy piezo-ceramic transducers, IEEE Trans. Ultrason. Ferroelect. Freq. Contr. 44 (1) (1997), pp. 60-66.

15. W.M. Leach, Controlled-source analogous circuits and SPICE models for piezoelectric transducers. IEEE Trans. Ultrason. Ferroelect. Freq. Contr. 41 (1) (1994), pp. 60-66.

16. A. Ramos, J.L. San Emeterio, P.T. Sanz. Dependence of Pulser Driving Responses on Electrical and Motional Characteristics of NDE Ultrasonic Probes. Ultrasonics Vol. 38 (1-8). pp. 553-558. April 2000. DOI: 10.1016/S0041-624X(99)00072-4.

17. M.L. Lethiecq, L.P. Tran-Huu-Hue, F. Patat, L. Pourcelot. Measurements of losses in five piezoelectric ceramics between 2 and 50 MHz, IEEE Trans. Ultrason. Ferroelect. Freq. Contr. 40 (3) (1993) 232-237

18. A. Ramos, A. Ruiz, J.L. San Emeterio, P.T. Sanz. Pspice circuital modeling of ultrasonic imaging transceivers including frequencydependent losses and signal distortions in electronic stages. Ultrasonics, Vol. 44, nº s1, 2006, pp. 995-1000.

19. J.L. San Emeterio, A. Ramos. Models for piezoelectric transducers used in broadband ultrasonic applications. Chapter 3 in book: "Piezoelectric Transducers and Applications". Berlin, New York, 2004. Springer-Verlag

20. J.L San Emeterio, P.T. Sanz, E. Riera, A. Ramos. Una implementación del modelo KLM para transductores piezoeléctricos en modo espesor. Anales Fisica B. 84:11-19, 1988.

21. L. Svilainis, A. Chaziachmetovas, V. Dumbrava. Efficient high voltage pulser for piezoelectric air coupled transducer. Ultrasonics 51(1):225-231. June 2012. DOI 10.1016/j.ultras.2012.06.004

22. $\mathrm{Yu} \mathrm{Xu,} \mathrm{Wei} \mathrm{Chen} \mathrm{et} \mathrm{al..} \mathrm{Megahertz} \mathrm{high} \mathrm{voltage} \mathrm{pulse} \mathrm{generator} \mathrm{suitable} \mathrm{for} \mathrm{capacitive} \mathrm{load.} \mathrm{AIP} \mathrm{Advances} \mathrm{7(11):115210.} 2017$. DOI: $10.1063 / 1.5006827$. 